\title{
Features of schemes of the explosive loosening, with big slowdowns
}

\author{
Alexandr Leshchinskiy ${ }^{1 *}$, Evgeniy Shevkun ${ }^{1}$, Yuriy Lysak ${ }^{2}$ and Andrey Plotnikov ${ }^{2}$ \\ ${ }^{1}$ Pacific State University, Chair of Transport and Technological Systems in Construction and Mining, \\ 680035 Khabarovsk. Russian Federation; \\ 2"AVT-Amur" Limited Liability Company, 675000, Blagoveshchensk city, Russia
}

\begin{abstract}
The task of the article is to determine the influence of the blasting charge power on the intensity of preliminary destruction of the blasting block mass at long delays. Increase of the destruction radius $r$ from the blasthole charges action at diagonal pattern of hole-by-hole blasting, caused by change of explosive charge energy or rock properties, decreases the number of stress waves passing in the vicinity of specific blast holes. They cause the rock disturbance in the pre-destruction area due to increasing of explosion energy absorption in the area of blasthole charges destruction. However, at the same time, the value of the predestruction factor increases. The calculation shows that 3.5 times increase of the blasthole charge destruction radius, reduces in 1.36 times the number of stress waves passing through the vicinity of specific blasthole charges, but the factor of pre-destruction intensity increases tenfold and the total impact of these factors - by a factor of 8 .
\end{abstract}

\section{Introduction}

Blasting rock destruction is characterized by one type of destruction - separation under the tensile stresses action from the compression wave action in the rarefaction phase. This leads to formation of a fracture system cutting the rock mass [1,2]. The qualitative indices of explosions in the quarries of Navoi Mining and Metallurgical Plant using non-electric initiation systems of the ISKRA type are characterized by the compact form of disintegration of the exploded rock mass, which contributes to losses and dilution reduction; the decrease of the coarse-grained rock yield; improvement of the quality of the ledge bottom finalization and seismic effect decrease. The improvement of the above mentioned parameters is explained by multiple explosive loading of the rock mass during hole by hole blasting with long delays (150 ms and more), which contributes to the formation of additional exposed cuts, increasing the impact of blasted rock flows.

In recent years, many complex problems were successfully solved, thanks to greater attention of scientists, engineers and practitioners, engaged in blasting, to the problems of physical foundations of industrial explosions in rocks, to technology improving and creation engineering methods for explosion energy control. One of the research trends includes short-delayed blasting, blasting without a compensatory slot, with interhole slowing down,

*Corresponding author:000399@pnu.edu.ru 
etc. Thus, the task has been put to determine the blasting charge power impact on intensity of preliminary destruction of an explosive block mass.

\section{Methods}

The process of blasting rock fragmentation has been studied by many scientists. Taking into account all achieved, the effect of the explosion on the rocks is considered as fragile destruction of the elastic plane medium - a plane problem, the solution of which is brought to definition of the required explosive ratio [3]. Since the length of the charge exceeds its diameter by several orders of magnitude and the rock shifts caused by the borehole charge have axial symmetry, the character of the charge action nature is suggested to determine by solving a plane (two-dimensional) task.

The stress wave extends beyond the explosive cavity boundaries, carrying out the predestruction of the holes surroundings. The magnitude of deformation (predestruction) of the holes surroundings will be determined by the formula

$$
\varepsilon_{i}=\varepsilon_{\max } K_{i}=\varepsilon_{\max }\left(\frac{r}{R_{i}}\right)^{2},
$$

where $\varepsilon_{i}$ is the deformation value of the $i$-th hole surroundings; $K_{i}$ is the factor of predestruction intensity, taking into account the change of deformation value with distance from the axis of the blasting hole; $r$ is the radius of hole destruction zone; $R_{i}$ is the distance from the axis of the $i$-th borehole to the blasting hole.

Factor $K_{i}$ is equal to 1 when the maximum deformation of the blast cavity of the hole $\varepsilon_{\max }$ is reached, i.e. the blast cavity of the hole is completely destroyed.

For example, let's consider a diagonal blasting pattern. We use the grapho-analytical method to determine the intensity of preliminary destruction of the blasting holes surroundings.

The radius of the hole destruction zone $r$ can reach the limit value of 40 charge radii $\left(\mathrm{R}_{\mathrm{c}}\right)$ and is an indirect expression of the explosive charge power of the blasting hole. The network of holes is $4 \times 4 \mathrm{~m}$ (Fig. 1). Its number is shown above the hole in the order of blasting; under the hole - the value of delay after the explosion of the first hole, in ms.

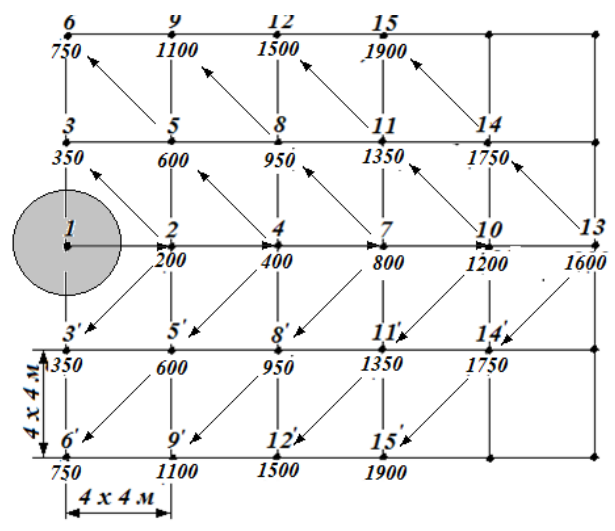

$a$

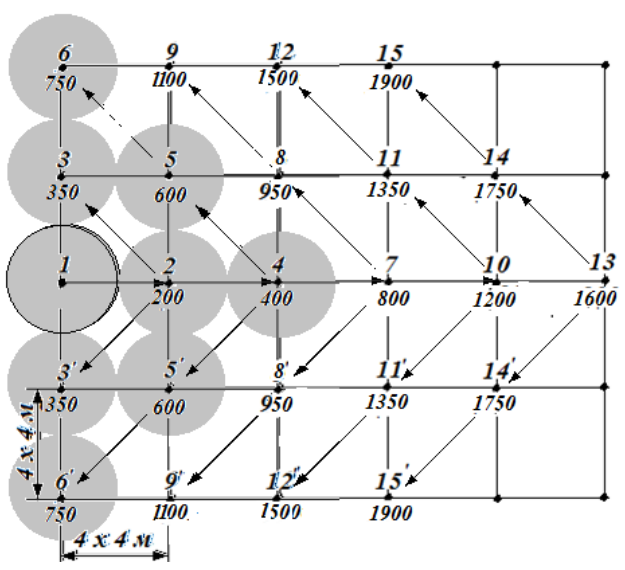

$b$

Fig. 1. Patterns of development of predestruction zone of the block with diagonal hole by hole blasting and initiating the surface network from the end hole in the third row of block: $a$ - after the explosion of the first hole; $b$ - after the explosion of the eighth hole

To determine the impact of the explosive charge power on the predestruction of the rock mass, we consistently take the radius $r$ equal to $1.0,2.0,2.5,3.0,3.5 \mathrm{~m}$. 
A non-electric system, such as RIONEL, was used for blasting. Delay between the holes of the surface network was made by RIONEL X device: in a row - $200 \mathrm{~ms}$, between rows $150 \mathrm{~ms}$. In Fig. 2, $a$, the patterns of development of the block predestruction zone with diagonal hole by hole blasting and initiation of the surface network from the end hole in the third row of the block at $\mathrm{r}=1.0 \mathrm{~m}$ are shown. Above the hole there is its number in the explosion order; under the hole there is the value of total factor $\boldsymbol{K}_{i}$ from explosion of neighboring holes.

The process is symmetrical with regard to the middle row at the diagonal blasting pattern, so it is enough to consider the destruction intensity of the surroundings from 1 st to 15 th holes.

In Table 1 it can be seen that $\boldsymbol{K}_{\boldsymbol{i}}$ factors do not reach 0.5 , i.e. half of the limit value of deformation $\varepsilon_{\max }$, and the rock in the vicinity of these holes requires additional blasting.

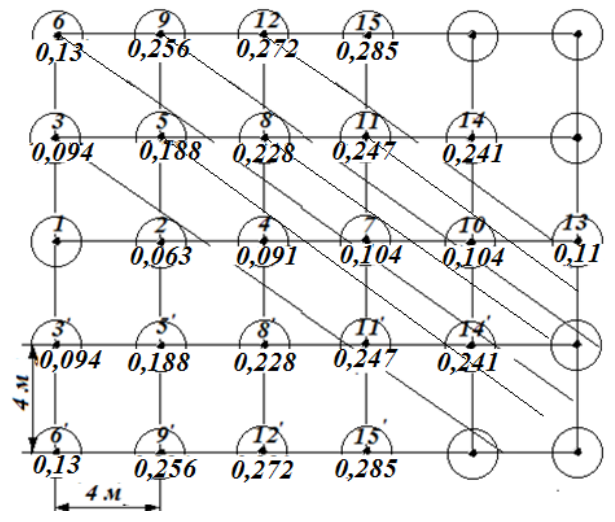

$a$

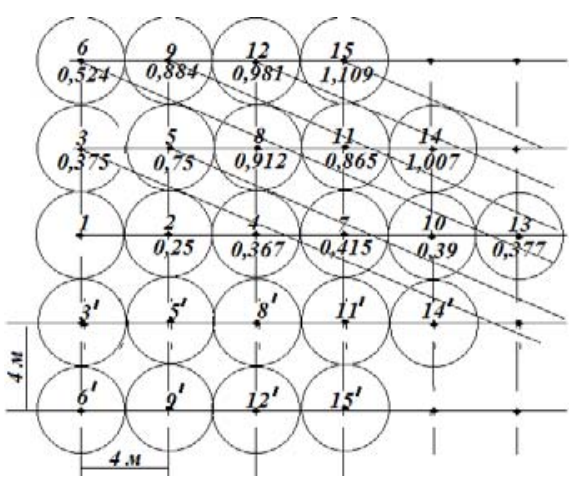

$b$

Fig. 2. Patterns of development of the block predestruction zone with diagonal hole-by-hole blasting and initiation of the surface network from the end hole in the third row of the block: $a-$ at $r=1.0 \mathrm{~m} ; b$ - at $r=2.0 \mathrm{~m}$

Table 1. Values of the predestruction intensity factor $\boldsymbol{K}_{\boldsymbol{i}}$ in vicinity of $2-15$ holes at $r=1.0 \mathrm{~m}$

\begin{tabular}{|c|c|c|c|c|c|c|c|c|c|c|c|c|c|c|c|c|}
\hline \multirow{16}{*}{ 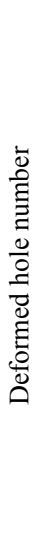 } & & \multicolumn{15}{|c|}{ Blasting hole number } \\
\hline & & 1 & 2 & 3 & 4 & 5 & 6 & 7 & 8 & 9 & 10 & 11 & 12 & 13 & 14 & $\Sigma K_{u}$ \\
\hline & 2 & 0,063 & & & & & & & & & & & & & & 0,063 \\
\hline & 3 & 0,063 & 0,031 & & & & & & & & & & & & & 0,094 \\
\hline & 4 & 0,016 & 0,063 & 0,012 & & & & & & & & & & & & 0,091 \\
\hline & 5 & 0,031 & 0,063 & 0,063 & 0,031 & & & & & & & & & & & 0,188 \\
\hline & 6 & 0,016 & 0,012 & 0,063 & 0,008 & 0,031 & & & & & & & & & & 0,13 \\
\hline & 7 & 0,007 & 0,016 & 0,006 & 0,063 & 0,012 & & & & & & & & & & 0,104 \\
\hline & 8 & 0,012 & 0,031 & 0,016 & 0,063 & 0,063 & 0,012 & 0,031 & & & & & & & & 0,228 \\
\hline & 9 & 0,012 & 0,016 & 0,031 & 0,012 & 0,063 & 0,063 & 0,031 & 0,031 & & & & & & & 0,256 \\
\hline & 10 & - & 0,007 & - & 0,016 & 0,006 & - & 0,063 & 0,012 & - & & & & & & 0,104 \\
\hline & 11 & 0,006 & 0,012 & 0,007 & 0,031 & 0,016 & 0,006 & 0,063 & 0,063 & 0,012 & 0,031 & & & & & 0,247 \\
\hline & 12 & 0,008 & 0,012 & 0,012 & 0,016 & 0,031 & 0,016 & 0,012 & 0,063 & 0,063 & 0,008 & 0,031 & & & & 0,272 \\
\hline & 13 & - & - & 0,006 & 0,007 & - & - & 0,016 & 0,006 & - & 0,063 & 0,012 & & & & 0,11 \\
\hline & 14 & - & 0,006 & - & 0,006 & 0,007 & - & 0,031 & 0,016 & 0,006 & 0,063 & 0,063 & 0,012 & 0,031 & & 0,241 \\
\hline & 15 & - & 0,008 & 0,006 & 0,012 & 0,012 & 0,007 & 0,016 & 0,031 & 0,016 & 0,012 & 0,063 & 0,063 & 0,008 & 0,031 & 0,285 \\
\hline
\end{tabular}

The rock mass destroyed by previous explosions prevents the passage of stress waves generating deformations. For example, in case of hole 9 explosion, the zone of destruction created by hole 8 explosion absorbs stress waves in the direction of hole 10 . 
Fig. 2, $b$ shows the patterns of development of the block predestruction zone with diagonal hole-by-hole blasting and initiation of the surface network from the end hole in the third row of the block at $r=2.0 \mathrm{~m}$.

Table 2 shows the values of $\boldsymbol{K}_{i}$ with diagonal hole-by-hole blasting at $r=2.0 \mathrm{~m}$. With increase of explosive charge power up to the value allowing to increase the destruction radius from $r=1.0 \mathrm{~m}$ up to $r=2.0 \mathrm{~m}$, i.e. twice, it is seen, that the factors $K_{i}$ of two from 14 holes $(14$ and 15$)$ are more than 1 , and of four holes $(8,9,11,12)$ are close to 1 , and the rock in these holes surroundings is destroyed or requires additional blasting by small explosive charge. The increase of the destruction radius $r$ of the blasting hole has somewhat reduced the area of the massif predestruction compared with previously considered variant. Thus, the number of deformation waves repeated passes decreased by 5 due to their absorption by the loosened rock mass.

Table 2. Values of the factor $\boldsymbol{K}_{i}$ of $2-15$ holes, $r=2.0 \mathrm{~m}$

\begin{tabular}{|c|c|c|c|c|c|c|c|c|c|c|c|c|c|c|c|c|}
\hline \multirow{16}{*}{ 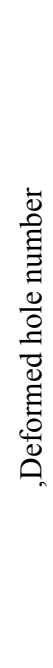 } & & \multicolumn{15}{|c|}{ Blasting hole number } \\
\hline & & 1 & 2 & 3 & 4 & 5 & 6 & 7 & 8 & 9 & 10 & 11 & 12 & 13 & 14 & $\Sigma K_{i}$ \\
\hline & 2 & 0,25 & - & - & - & - & - & - & - & - & - & - & - & - & - & 0,25 \\
\hline & 3 & 0,25 & 0,125 & - & - & - & - & - & - & - & - & - & - & - & - & 0,375 \\
\hline & 4 & 0,062 & 0,25 & 0,05 & - & - & - & - & - & - & - & - & - & - & - & 0,367 \\
\hline & 5 & 0,125 & 0,25 & 0,25 & 0,125 & - & - & - & - & - & - & - & - & - & - & 0,75 \\
\hline & 6 & 0,062 & 0,05 & 0,25 & 0,037 & 0,125 & - & - & - & - & - & - & - & - & - & 0,524 \\
\hline & 7 & 0,028 & 0,062 & 0,025 & 0,25 & 0,05 & - & - & - & - & - & - & - & - & - & 0,415 \\
\hline & 8 & 0,05 & 0,125 & 0,062 & 0,25 & 0,25 & 0,05 & 0,125 & - & - & - & - & - & - & - & 0,912 \\
\hline & 9 & 0,05 & 0,062 & 0,125 & 0,05 & 0,25 & 0,25 & 0,037 & 0,06 & - & - & - & - & - & - & 0,884 \\
\hline & 10 & - & 0,028 & - & 0,062 & 0,025 & - & 0,25 & 0,025 & - & - & - & - & - & - & 0,39 \\
\hline & 11 & 0,025 & 0,05 & 0,028 & 0,125 & 0,062 & 0,025 & 0,25 & 0,25 & 0,025 & 0,025 & - & - & - & - & 0,865 \\
\hline & 12 & 0,037 & 0,05 & 0,05 & 0,062 & 0,125 & 0,062 & 0,05 & 0,25 & \begin{tabular}{|l}
0,25 \\
\end{tabular} & \begin{tabular}{|l|}
0,02 \\
\end{tabular} & 0,025 & - & - & - & 0,981 \\
\hline & 13 & - & - & - & 0,028 & - & - & 0,062 & 0,012 & - & 0,25 & 0,025 & - & - & - & 0,377 \\
\hline & 14 & - & 0,025 & - & \begin{tabular}{|l|}
0,05 \\
\end{tabular} & 0,028 & - & 0,125 & \begin{tabular}{|l|}
0,062 \\
\end{tabular} & - & - & \begin{tabular}{|l}
0,25 \\
\end{tabular} & 0,025 & \begin{tabular}{|l|}
0,25 \\
\end{tabular} & - & 1,077 \\
\hline & 15 & - & 0,037 & 0,025 & 0,05 & 0,05 & 0,028 & 0,062 & 0,125 & 0,062 & 0,025 & 0,25 & 0,25 & 0,02 & 0,125 & 1,109 \\
\hline
\end{tabular}

Further, to determine the influence of explosive charge power on the rock mass predestruction, we increase the radius of the blasting hole destruction zone up to $2.5 \mathrm{~m}$ (Fig. 3, $a$, Table 3). With the increase of the destruction radius from $r=2.0 \mathrm{~m}$ to $r=2.5 \mathrm{~m}$, i.e. by 1.25 times, the factors of relative deformation of the surroundings of $6-14$ holes $(8,9,11$, 12.14 and 15) are greater than 1 , and the rock in the vicinity of these holes is destroyed and does not require additional explosion. The increase of the destruction radius $r$ of a blasting hole from $2.0 \mathrm{~m}$ to $2.5 \mathrm{~m}$ has somewhat reduced the predestruction area of the massif in comparison with the destruction radius $r$ of a blasting hole. Thus, the number of repeated passages of deformation waves decreased by 5 due to their damping by loosened rock mass.

At the next stage we increase the radius of the blasting hole destruction zone up to $3.0 \mathrm{~m}$ (Fig. 4, Table 4). The factors of relative deformation in the vicinity of three of 14 holes (9, 12 and 15$)$ are more or equal to 2 , four holes $(5,8,11,14)$ - from 1.5 to 1.8 , one hole $(6)-$ close to 1 , the rock in the vicinity of these holes was destroyed. Factors of relative deformation of six holes $(2,3,4,7,10$ and 13) are within the $0.56-0.82$ range, the rock in the surroundings of these holes requires additional blasting with a small explosive charge. 


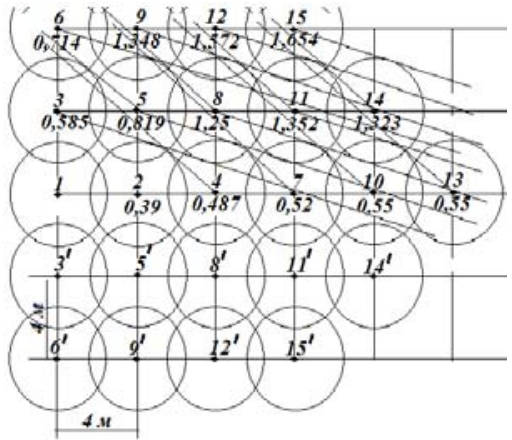

$a$

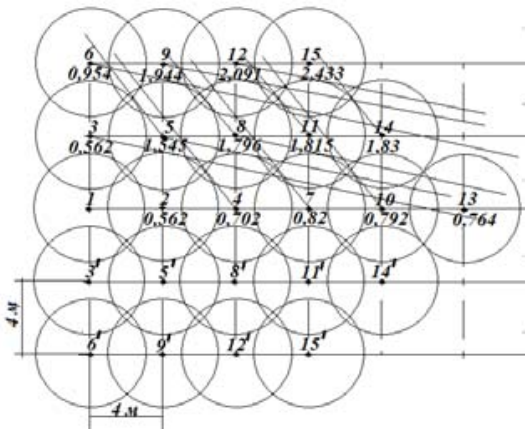

$b$

Fig. 3. Patterns of block predestruction zone development: $a$ - at $r=2.5 \mathrm{~m} ; b-$ at $r=3.0 \mathrm{~m}$

Table 3. Values of factor $K_{i}$ from 2 to 15 holes

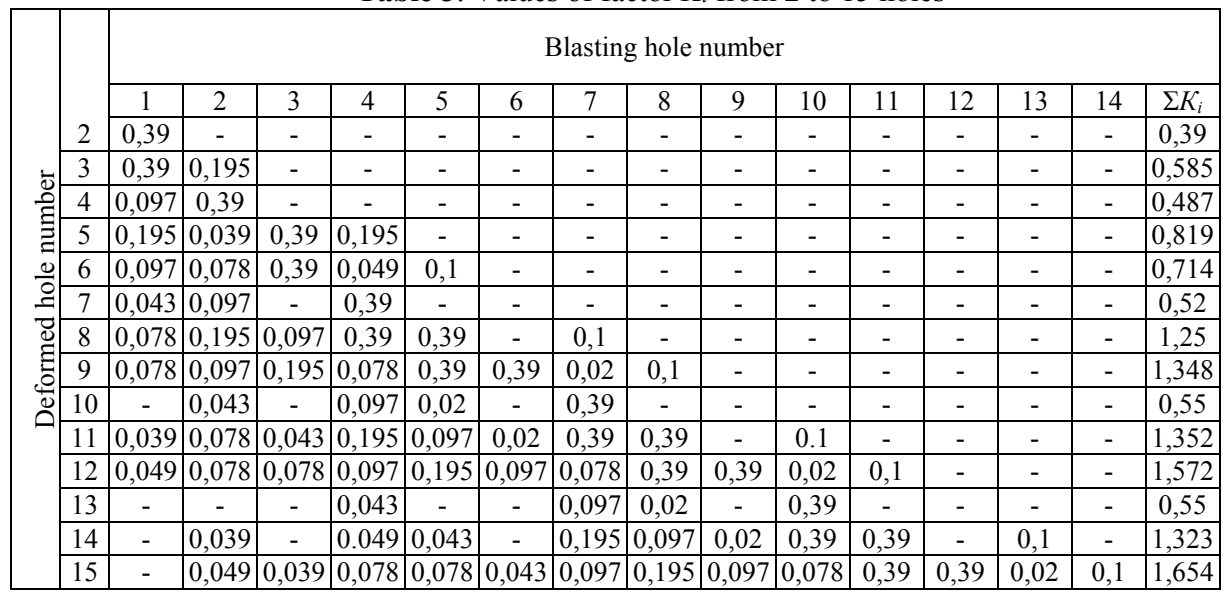

The number of repeated passages of deformation waves decreased by 7 due to their damping by loosened rock mass (Table 6).

At the last stage we increase the radius of blasting hole destruction zone up to $3.5 \mathrm{~m}$ (Fig. 4, $b$, Table 5). Factors of relative deformation in the vicinity of three of 14 holes $(9,12$ and 15$)$ are more than 2 , four holes $(5,8,11,14)$ - from 1.5 to 1.8 , one hole $(6)$ - close to 1 , the rock in these holes surroundings was destroyed. Relative deformation factors of six holes $(2,3,4,7,10$ and 13$)$ are within the range of $0.56-0.82$, the rock in the vicinity of these holes requires additional explosion with a small explosive charge.

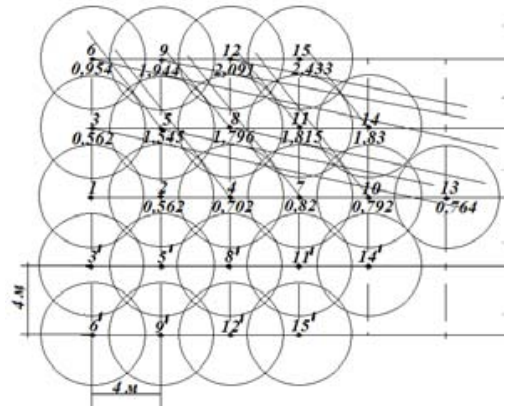

$a$

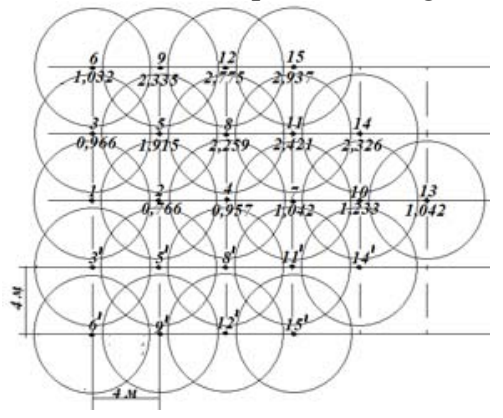

$b$

Fig. 4. Patterns of block predestruction zone development $a-$ at $r=3.0 \mathrm{~m} ; b-$ at $r=3.5 \mathrm{~m}$ 
Table 4. Values of $K_{i}$ factor from 2 to 15 holes, $r=3.0 \mathrm{~m}$

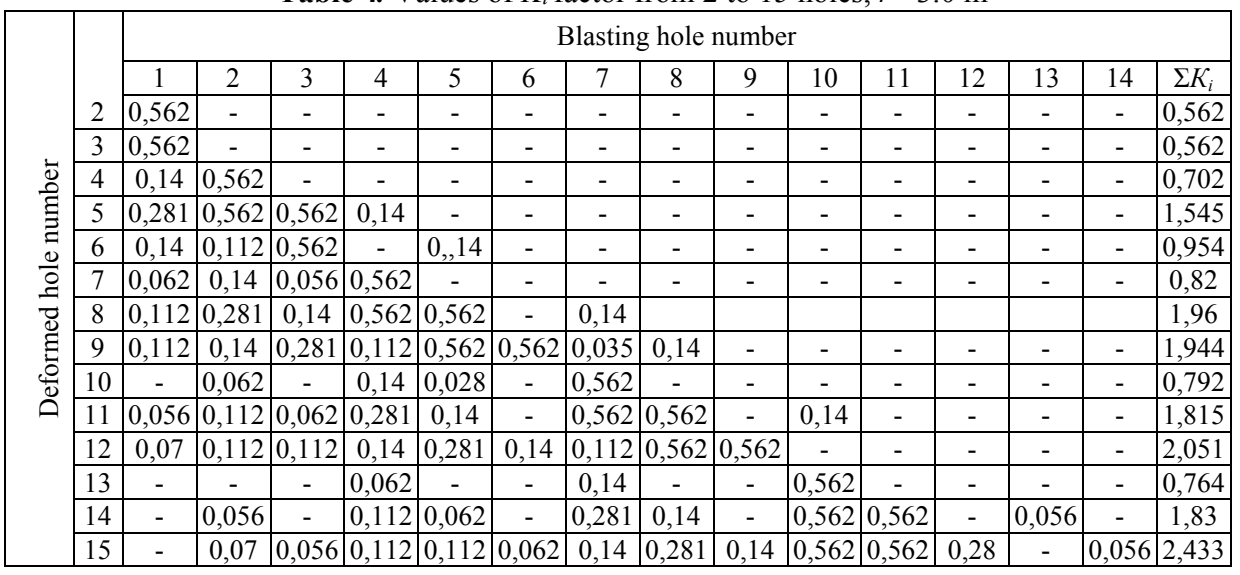

The increase of the destruction radius $r$ of the blasting hole up to $3.5 \mathrm{~m}$ reduced by 11 the number of predestructions of the rock mass in comparison with the $3.0 \mathrm{~m}$ destruction radius of the blasting hole (Table 6).

Table 5. Values of $K_{i}$ factor from 2 to 15 holes, $r=3.5 \mathrm{~m}$

\begin{tabular}{|c|c|c|c|c|c|c|c|c|c|c|c|c|c|c|c|c|}
\hline & \multicolumn{15}{|c|}{ Blasting hole number } \\
\hline & & 1 & 2 & 3 & 4 & 5 & 6 & 7 & 8 & 9 & 10 & 11 & 12 & 13 & 14 & $\Sigma K_{i}$ \\
\hline & 2 & 0,766 & - & - & - & - & - & - & - & - & - & - & - & - & - & 0,766 \\
\hline & 3 & 0,766 & 0,2 & - & - & - & - & - & - & - & - & - & - & - & - & 0,966 \\
\hline & 4 & 0,191 & 0,766 & - & - & - & - & - & - & - & - & - & - & - & - & 0,957 \\
\hline है & 5 & 0,383 & 0,766 & 0,766 & - & - & - & - & - & - & - & - & - & - & - & 1,915 \\
\hline 吾 & 6 & 0,191 & 0,076 & 0,766 & - & - & - & - & - & - & - & - & - & - & - & 1,032 \\
\hline$\frac{0}{0}$ & 7 & 0,085 & 0,191 & - & 0,766 & - & - & - & - & - & - & - & -- & - & - & 1,042 \\
\hline 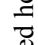 & 8 & 0,153 & 0,383 & 0,191 & 0,766 & 0,766 & - & - & - & - & - & - & - & - & - & 2,259 \\
\hline छ & 9 & 0,153 & 0,191 & 0,383 & 0,076 & 0,766 & 0.766 & - & - & - & - & - & - & - & - & 2,335 \\
\hline 륭 & 10 & - & 0,085 & - & 0,191 & - & 0,191 & 0,766 & - & - & - & - & - & - & - & 1,233 \\
\hline & 11 & 0,076 & 0,153 & 0,085 & 0,383 & 0,191 & - & 0,766 & 0,766 & - & - & - & - & - & - & 2,421 \\
\hline & 12 & 0,096 & 0,153 & 0,153 & 0,191 & 0,383 & 0,191 & 0,076 & 0,766 & 0,766 & - & - & - & - & - & 2,775 \\
\hline & 13 & - & 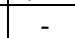 & 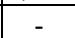 & 0,085 & 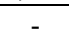 & - & 0,191 & - & - & \begin{tabular}{|l|}
0,766 \\
\end{tabular} & - & - & - & - & 1,042 \\
\hline & 14 & - & 0,076 & - & 0,153 & 0,085 & - & 0,383 & 0,096 & - & 0,766 & 0,766 & - & - & - & 2,326 \\
\hline & 15 & - & 0,096 & 0,076 & 0,153 & 0,153 & 0,085 & 0,191 & 0,383 & 0,191 & 0,076 & 0,766 & 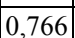 & - & - & 2,037 \\
\hline
\end{tabular}

Thus, as the power of the explosive charge increases (in this case, increase of radius $r$ of the destruction zone of a blasting hole), the total factor of preliminary destruction of the block holes vicinity $\boldsymbol{\Sigma} \boldsymbol{K}_{\boldsymbol{i}}$ increases (Fig. 5).

Analysis of Fig. $5 b$ shows that the number of stress waves passages through the vicinity of the block holes with increasing radius $r$ of the destruction zone of the block blasting holes decreases. It is explained by the following: at more powerful explosions the large area of the blasting block is loosened up and the stress waves are absorbed by loosened rock mass. 


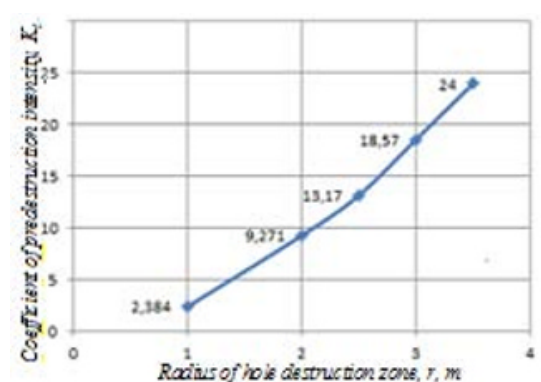

$a$

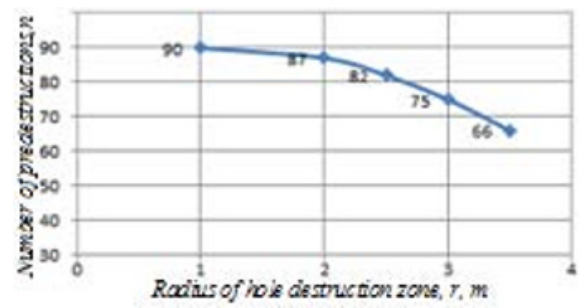

$b$

Fig. 5. Dependence of the values of the total intensity factor of preliminary destruction of the block holes vicinity $\Sigma K_{i}(a)$ and the number of deformation waves passing through the surroundings of the block holes $(b)$ from the radius $r$ of the destruction zone of blasting holes in the block with diagonal hole-by-hole blasting

\section{Conclusions}

The increase of the destruction radius $r$ from the borehole charges action in a diagonal holeby-hole blasting pattern, caused by change of explosive charge power or rocks properties, reduces the number of stress waves passing through the vicinity of the certain blasting holes, causing the rocks disturbance in the predestruction zone, due to increasing absorption of the explosion energy in the zone of blasthole charge destruction. However, at the same time, the value of the predestruction factor increases. The calculation shows that a 3.5 -fold increase in the destruction radius of a blasthole charge reduces the number of stress waves passing through the vicinity of specific hole charges by 1.36 times, but the predestruction intensity coefficient increases by a factor of 10 and the total impact of these factors - by a factor of 8 .

\section{References}

1. N.P. Snitka, I. T. Misliboev, Yo.L. Karimov, Mining Journal of Uzbekistan, 2/61, 7-11. (2015) (in Russian)

2. Xiaodong Fu, Qian Sheng, Yonghui Zhang, Jian Chen, International Journal of Rock Mechanics and Mining Sciences. 80, 155-170 (2015)

3. N.G. Shtukarin, Physics of explosions, 309 (2010) (in Russian) 